\title{
Simple and Associative Recognition Memory in the Hippocampal Region
}

\author{
Craig E.L. Stark ${ }^{1}$ and Larry R. Squire ${ }^{2,3}$ \\ ${ }^{1}$ Department of Psychiatry, University of California San Diego, San Diego, California 92093, USA; ${ }^{2}$ Veterans Affairs Medical Center, \\ San Diego, California 92161, USA; ${ }^{3}$ Departments of Psychiatry, Neurosciences, and Psychology, University of California San Diego, \\ San Diego, California 92093, USA
}

\begin{abstract}
Although it is well established that the hippocampal region is involved in the formation of declarative memory, the exact nature of its involvement is unclear. One view is that the hippocampal region is involved only in tasks that require the formation or use of associations. According to this view, the hippocampal region is not involved in traditional tests of recognition memory. An alternative view is that the hippocampal region combines and extends the processing carried out by structures in the parahippocampal gyrus and that it is involved in all forms of declarative memory, including recognition memory. Using event-related functional magnetic resonance imaging (fMRI), we observed hippocampal activity during both traditional and associative recognition memory tasks. Critically, the hippocampal region was no more active in the associative recognition task than in the traditional recognition task.
\end{abstract}

Studies of humans and experimental animals have identified a system of medial temporal lobe structures essential for the formation of declarative memory (memory for facts and events) (Milner et al. 1998). The contributions of the separate components of this system (the entorhinal, perirhinal, and parahippocampal cortices and the hippocampal region) are not well understood. It has been suggested that the hippocampal region is essential for episodic memory and for other tasks requiring the formation or use of associations, but that it is not involved in recognition memory tasks (at least when they do not involve a recollective or associative component) (Henke et al. 1997, 1999; Mishkin et al. 1997; Vargha-Khadem et al. 1997; Aggleton and Brown 1999; Eldridge et al. 2000; Brown and Aggleton 2001).

An alternative view is that the hippocampal region combines and extends the processing carried out by structures in the parahippocampal gyrus and that it is involved in all forms of declarative memory (Stark and Squire 2000a; Suzuki and Eichenbaum 2000; Zola and Squire 2000). According to this view, although differences in the anatomical connections and computational properties of the structures within the medial temporal lobe may correspond to differences in function (e.g., the perirhinal cortex might be more important for visual memory and the parahippocampal cortex might be more important for spatial memory. See Zola and Squire 2000; Zola-Morgan and Squire 1993), these differences are not absolute. Functional magnetic resonance imaging (fMRI) can be used to provide converging evidence about the role of the hippocampal region in declarative

${ }^{3}$ Corresponding author.

E-MAIL 1squire@ucsd.edu; FAX (858) 552-7457.

Article and publication are at http://www.learnmem.org/cgi/doi/ $10.1101 / \mathrm{lm} .40701$. memory. After numerous initial failures, investigators have observed activity related to recollective success in the hippocampal region during recognition memory testing (Gabrieli et al. 1997; Eldridge et al. 2000; Stark and Squire 2000a,b). Only one of these four studies used a traditional recognition memory test in which participants made simple yes/no judgments about whether a stimulus was recognized as being one of the previously studied stimuli (Stark and Squire 2000a). The other three studies used nontraditional recognition memory tasks. Two of the studies (Gabrieli et al. 1997; Stark and Squire 2000b) involved a cross-modal task (participants study objects and were tested with words that named or did not name the objects). This task required the use of an association during the recognition test and may place greater emphasis on episodic or recollective components of recognition memory. The third study (Eldridge et al. 2000) involved a two-stage "Remember-Know" procedure that attempts to isolate hippocampal activity related to explicit recollection (Tulving 1985). In the only study that used a traditional recognition memory task (Stark and Squire 2000a), activity was observed in the hippocampal region both when objects were used at study and test and when words were used at study and test. Thus, hippocampal activity has been observed during both traditional and nontraditional recognition memory tests.

It has been suggested that the fMRI data support the view that the hippocampus is more active when the recognition task requires an association, as in the cross-modal association task described above (Brown and Aggleton 2001). However, this issue is difficult to settle using between-study comparisons, and it has not yet been explored with fMRI using the appropriate within-study comparison. Here, we used rapid event-related fMRI (Dale and Buckner

LEARNING \& MEMORY 8:190-197 @ 2001 by Cold Spring Harbor Laboratory Press ISSN1072-0502/01 \$5.00

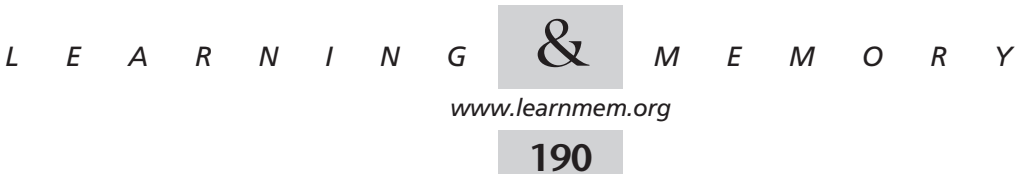


1997; Dale 1999) to assess hippocampal activity during both traditional and nontraditional recognition tests. Specifically, we examined activity in the hippocampal region associated with recollective success (Hits vs. Correct Rejections) during three different recognition memory tasks. In one task (Object-Object), pictures of nameable objects were presented both at study and during the recognition memory test. In a second task (Word-Word), words (names of objects) were presented both at study and during the recognition memory test. In a third task (Object-Word), an associative recognition task was administered in which pictures of nameable objects were presented during study, and words (names of objects) were presented during the recognition memory test. All three tasks were administered to each participant. In addition, each recognition memory task was administered three times in succession using the same stimuli to examine the effect of test repetition.

In all three recognition tasks, activity related to recol- lective success (Hits vs. Correct Rejections) was observed in the hippocampal region. The critical comparison to determine whether the hippocampal region was more active during the associative recognition memory task than during the traditional recognition memory task is to contrast the Object-Object and Object-Word tasks, as both tasks assess recognition memory for a studied object. We found no indication that the hippocampal region was more active in the associative recognition task than in a traditional recognition task.

\section{RESULTS}

Across the three recognition tests in the Object-Object condition, participants were $92.9 \%$ correct (range, $92.3 \%-93.7 \%$ ). The mean hit rate was $73.4 \%$, and the mean false alarm rate was $7.0 \%$. Across the three recognition tests in the Word-Word condition, participants were $84.4 \%$ correct (range, $82.7 \%-86.1 \%$ ). The mean hit rate was $66.9 \%$, and the mean false alarm rate was $13.1 \%$. Finally, across the three recognition tests in the ObjectWord condition, participants were $90.6 \%$ correct (range, 90.0\%$91.0 \%$ ). The mean hit rate was $70.6 \%$, and the mean false alarm rate was $6.1 \%$. Overall recognition accuracy did differ across the three test conditions $(F(2,24)=$ $10.8, P<.001)$ with lower accuracy in the Word-Word condition than in the other two conditions $(P$ values $<.05)$. Recognition accuracy in the Object-Object condition was marginally greater $(2.3 \%)$ than in the Object-Word condition $(t(12)=2.1, P=.06)$.

Activity during the recognition memory task related to Hits (correct "yes" responses to studied items) versus Correct Rejections (correct "no" responses to unstudied items) is shown for all three task conditions in Figure 1. Activity outside of the hippocampal region related to Hits versus Correct Rejections is identified in Table 1 . In all three tasks, activity was observed in the hippocampal region. In the Object-Object condition, activity in the right hippocampal region was observed in the second (Fig. 1b) and third (data not shown) administration of the recognition memory test, though
Figure 1 Areas of significant fMRI signal change related to recollective success (Hits vs. Correct the location of available data which are shown as colored overlays on the averaged structural images (transformed to the atlas of Talairach and Tournoux 1988). ( $a$ and $b$ ) fMRI data from the right hippocampal region (26 right) during the first (a) and second $(b)$ administration of the recognition memory test when the participants saw objects at both study and test (Object-Object task). (c) fMRI data from the left hippocampal region (21 left) during the first administration of the recognition memory test when the same participants saw words at both study and test (Word-Word task). (d) fMRI data from the right hippocampal region (26 right) during the first administration of the recognition memory test when the same participants saw objects at study and words (names of objects) at test (Object-Word task). Activation of the hippocampal region was observed in all three recognition memory tasks.

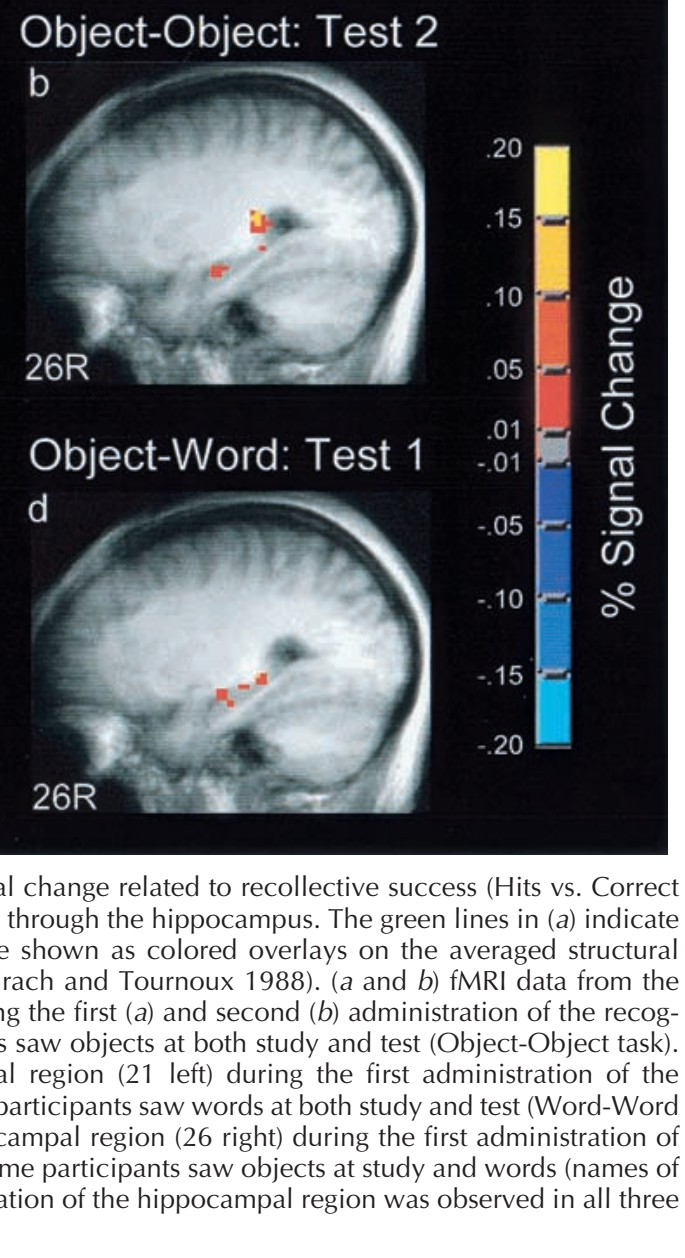


Table 1. Activity for Targets versus Foils Outside the Hippocampal Region

\begin{tabular}{|c|c|c|}
\hline Task & Hits $>$ Correct rejections & Hits $<$ Correct rejections \\
\hline $\begin{array}{l}\text { Object-Object } \\
\text { Test } 1\end{array}$ & $\begin{array}{l}\text { B precuneus, } B \text { intraparietal sulcus, } B \text { cuneus, } L \text { sup. } \\
\text { temporal g, } R \text { mid. temporal g, } L \text { insula. }\end{array}$ & L inf. temporal g, B fusiform g. \\
\hline $\begin{array}{l}\text { Object-Object } \\
\text { Test } 2\end{array}$ & $\begin{array}{l}\text { B precuneus, } B \text { cuneus, } L \text { mid. temporal g, } B \\
\text { cingualte, } L \text { parieto-occipital sulcus, } L \text { inf. parietal } \\
\text { lobule, } L \text { supra-marginal g, } L \text { transverse temporal g, } \\
L \text { putamen/globus pallidus, } R \text { caudate/hippocampal } \\
\text { tail, } R \text { parahippocampal g. }\end{array}$ & $\begin{array}{l}\text { B transverse temporal g, B mid. occipital } \\
\text { g, L parahippocampal g. }\end{array}$ \\
\hline Object-Object & $L$ cingulate, $B$ sup. temporal $g, B$ mid. temporal $g, R$ & L mid. occipital g, L putamen. \\
\hline Test 3 & cerebellum, L insula, L parahippocampal g. & \\
\hline Word-Word & B precuneus, B sup. temporal g., B cingulate, B inf. & \\
\hline Test 1 & parietal lobule, R pulvinar, B parahippocampal g. & \\
\hline $\begin{array}{l}\text { Word-Word } \\
\text { Test } 2\end{array}$ & $\begin{array}{l}\text { B sup. temporal g, } R \text { mid. temporal } g, B \text { inf. parietal } \\
\text { lobule, } R \text { pulvinar. }\end{array}$ & \\
\hline $\begin{array}{l}\text { Word-Word } \\
\text { Test } 3\end{array}$ & & \\
\hline Object-Word & B precuneus, $R$ mid. temporal $g$, $L$ inf. parietal lobule, & \\
\hline Test 1 & L putamen/globus pallidus, R pulvinar. & \\
\hline Object-Word & B precuneus, $R$ sup. temporal $g, L$ mid. temporal $g, R$ & L mid. temporal g. \\
\hline Test 2 & $\begin{array}{l}\text { fusiform, } \mathrm{R} \text { cingulate, } \mathrm{R} \text { inf. parietal lobule, } \mathrm{B} \\
\text { putamen/globus pallidus, } \mathrm{R} \text { lingual g. }\end{array}$ & \\
\hline Object-Word & & \\
\hline Test 3 & & \\
\hline
\end{tabular}

B, Bilateral; L, left; R, right; sup., superior; inf., inferior; mid., middle; g, gyrus.

not in the first. The absence of activity in the first test has been noted previously using this same task (Stark and Squire 2000a) and appears to follow from the high level of activity associated with encoding the novel foil objects. The finding is that there are high levels of activity associated with Correct Rejections (93\% of the foil items), and it is difficult to observe activity in a Hit versus Correct Rejection contrast (also see below).

In the Word-Word condition, activity was observed in the left hippocampal region during the first (but not the second or third) administration of the recognition memory test (Fig. 1c), also confirming previous findings (Stark and Squire 2000a). In the Object-Word condition, increased activity was observed in the right hippocampal region during the first (but not the second or third) administration of the recognition test (Fig. 1d). Previous studies using this task have reported either bilateral (Gabrieli et al. 1997) or right unilateral activity (Stark and Squire 2000b).

The question of interest concerned the level of hippocampal activity across tasks. Contrasting the level of activity across task conditions is made difficult by the observation that the encoding of novel stimuli into memory during the recognition memory test itself can produce activity in the hippocampal region (Stark and Squire 2000a). This enhanced activity, which results from encoding novel stimuli (such as the foil items that are correctly rejected in the first recognition memory test) can reduce the level of activity assessed by contrasts such as Hits versus Correct Rejections (or Targets vs. Foils), thereby resulting in a failure to observe activity (e.g., Fig. 1a). This effect is shown in the condition of the present study in Figure 2. Activity in the right hippocampal region associated with Correct Rejections was higher in the first recognition memory test (when these stimuli were novel) than in the second administration of the same recognition memory test. (Similar results were obtained when contrasting Correct Rejections in Test 1 vs. Test 3 and in Test 2 vs. Test 3 ). A comparison of the level of activity associated with Hits revealed no reliable differences in activity between any of the three administrations of the recognition memory test. Assessing activity in terms of Hits versus Correct Rejections, and contrasting this level of activity across the Object-Word and Object-Object conditions, is therefore inappropriate.

The critical comparison, therefore, is between the activity associated with Hits in the Object-Object and the Object-Word tasks. In both the Object-Object and Object-Word tasks, the study task is identical and memory for an object is being tested. The two tasks differ only in whether the stimulus itself (Object-Object) or the name of the stimulus (Object-Word) is used as the recognition memory cue. In the other two possible cross-task comparisons (Object-Object vs. Word-Word and Word-Word vs. Object-Word), the studied items that are being retrieved (words vs. objects) and the left/right locus of hippocampal activity differ across the two tasks. Accordingly, the activity associated with successfully recognizing an item cannot be compared across tasks in these two cases. It should be noted that the pattern of activity associated with Hits in a particular task will include not only activity associated with recollective success, but also activity associated with other components of the rec-

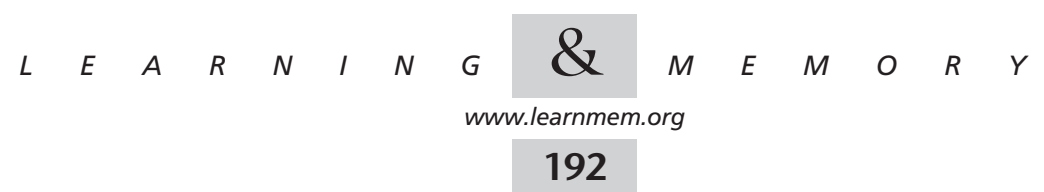




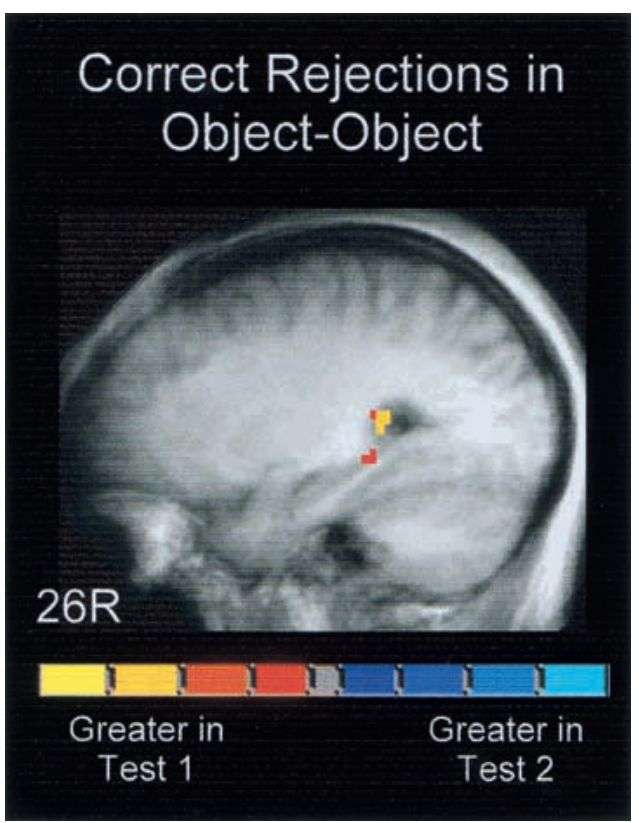

Figure 2 Areas of significant $f M R I$ signal change related to withinsubject contrasting of activity associated with trials in which there were Correct Rejections (correct "no" responses to unstudied items) between the first (Test 1) and the second (Test 2) administration of the recognition memory task in the Object-Object condition. The images are shown as a colored overlay on a sagittal section through the right hippocampal region (26 right). Greater activity in the right hippocampal region associated with Correct Rejections was observed in Test 1 relative to Test 2 . In Test 1 , the Correct Rejections were unfamiliar and likely resulted in hippocampal activity associated with memory encoding. In Test 2, these stimuli were now more familiar and less subject to an "encoding effect."

ognition process. To the extent that these other components are similar across tasks, contrasting Hits across tasks (rather than within a task) will remove the effects of these components.

Figure 3a shows areas in which the activity associated with Hits differed in the Object-Object and Object-Word tasks (first recognition test only). No differences in hippocampal activity were observed (only the right hippocampal region is illustrated). The results were the same when Hits were compared (Object-Object and Object-Word tasks) on the second and third recognition tests. Thus, successfully recognizing an object when the memory cue was a word did not result in any greater activity in the hippocampal region than successfully recognizing an object when the memory cue was the object itself.

Although the level of activity associated with Hits did not differ between the Object-Object and Object-Word tasks (Fig. 3a), the activity associated with Correct Rejections did differ (Fig. 3b). Specifically, activity in the right hippocampal region associated with Correct Rejections was higher in the first administration of the Object-Object task than in the first administration of the Object-Word task (but not in the second and third recognition tests). Because objects, but not words, are likely to be encoded in the right hippocampal region, this finding shows that during recognition memory testing the automatic encoding of unfamiliar stimuli (i.e., the foils in the first administration of the task) elicits increased activity in the hippocampal region that can reduce or eliminate activity as measured by a Hit versus Correct Rejection contrast (Stark and Squire 2000a).

\section{DISCUSSION}

Activity related to recollective success (Hits vs. Correct Rejections) was observed in the hippocampal region during all three recognition memory tasks. Activation was right unilateral when pictures of nameable objects were used at both study and test (Object-Object). Activation was left unilateral when words were used at both study and test (Word-Word). Activation was also right unilateral when pictures of nameable objects were used at study and when words were used at test (Object-Word). Consistent with previous results, both associative (Gabrieli et al. 1997; Stark and Squire 2000b) and traditional (Stark and Squire 2000a) recognition memory tasks elicited hippocampal activity.

When the critical comparison was made between the amount of activity associated with successfully recognized objects (Hits) in the Object-Object and Object-Word task conditions, no difference in activity in the hippocampal region was observed (Fig. 3a). Successfully recognizing an object in a traditional recognition memory task and successfully recognizing an object in an associative recognition memory task resulted in similar levels of hippocampal activity.

The results appeared at first to be different when activity in the two tasks was compared using a Hit versus Correct Rejection contrast (Fig. 1a versus 1d). That is, the Hit versus Correct Rejection contrast revealed hippocampal activity during the first administration of the Object-Word task, but did not reveal any activity during the Object-Object task. However, activity in the Object-Object task was absent only during the first administration of the recognition test, not during the second or third tests. Further, the apparent absence of activity during the first test in the Object-Object condition could be attributed to increased activity associated with Correct Rejections (Fig. 2 and Fig 3b) that was in turn due to the automatic encoding of the unfamiliar foil items. Thus, the observed difference in hippocampal activity between the Object-Word and Object-Object conditions (as measured in the Hit vs. Correct Rejection contrast) was an artifact of this encoding effect. The critical point is that activity associated with Hits did not differ between the Object-Word and Object-Object tasks across the three recognition tests.

Enhanced activity in the medial temporal lobe has been observed previously in association with the learning or encoding of unfamiliar stimuli (relative to familiar stimuli),

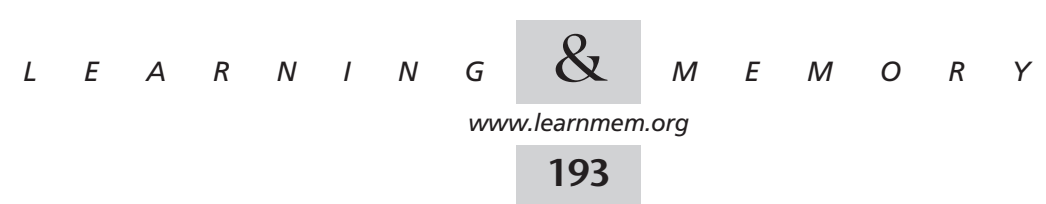




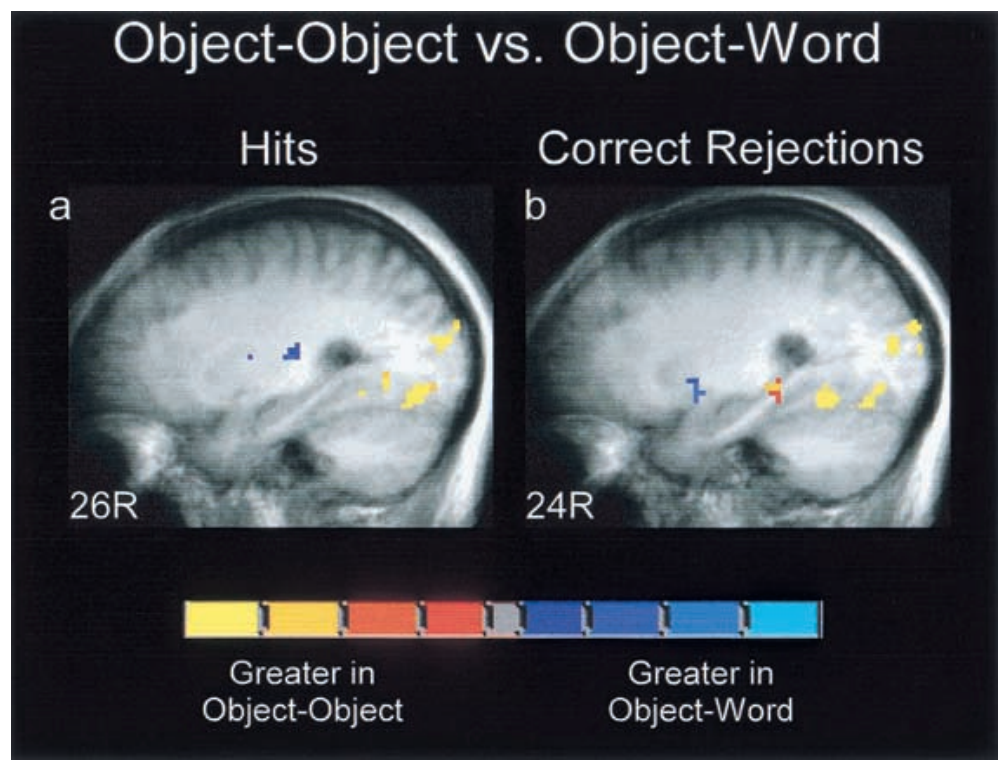

Figure 3 Areas showing significantly different activity associated with (a) Hits (correct "yes" responses) and (b) Correct Rejections (correct "no" responses) when contrasting the Object-Object and Object-Word conditions. The images are shown as colored overlays on sagittal sections through the right hippocampal region (26 right). Data from the first administration of the recognition memory test (Test 1 ) are shown. (a) Successfully recognizing an object in a traditional recognition memory task (Object-Object) and successfully recognizing an object in an associative recognition memory task (Object-Word) yielded no difference in hippocampal activation. (b) Greater activity associated with Correct Rejections was observed in the Object-Object task than in the Object-Word task. During the recognition memory test itself, the automatic encoding of unfamiliar stimuli (the foils in the ObjectObject task) elicited increased activity that reduced activity as measured by a Hit versus Correct Rejection contrast.

even when participants are instructed to view the stimuli passively (Stern et al. 1996; Lepage et al. 1998; Schacter and Wagner 1999). The activity is lateralized based on stimulus type. Activity is usually left unilateral during the encoding of words (Martin et al. 1997; Wagner et al. 1997; Kelly et al. 1998; but see Fernandez et al. 1998) and bilateral during the encoding of objects or pictures (Gabrieli et al. 1997; Martin et al. 1997; Brewer et al. 1998; Kelly et al. 1998). This relatively automatic activation of the medial temporal lobe associated with encoding unfamiliar stimuli works in direct opposition to finding a Hits versus Correct Rejection contrast during recognition memory testing, because in the first recognition test the Correct Rejections are unfamiliar. One explanation for previous failures to find hippocampal activation during recognition memory testing is that there may have been elevated activity in response to unfamiliar foils to such an extent that a target versus foil contrast could not be observed (cf. Eldridge et al. 2000; Haxby et al. 1996; also see Stark and Squire 2000a).

It has been suggested that the hippocampal region is needed only for associative recognition, not for traditional recognition tasks; or that the hippocampus supports the associative or recollective component of recognition memory (Henke et al. 1997, 1999; Mishkin et al. 1997; Vargha-Khadem et al. 1997; Aggleton and Brown 1999; Eldridge et al. 2000; Brown and Aggleton 2001). Although the correlational nature of fMRI data cannot determine whether a structure is necessary for a given task, the present findings argue against such a simple division of labor within the medial temporal lobe. By cuing memory for an object by presenting a word, the Object-Word task introduces an overtly associative component to the recognition task. In addition, in comparison to the traditional Object-Object recognition task (which can be performed on the basis of familiarity alone), the cross-modal Object-Word task places greater emphasis on the recollective component of recognition, as the same stimulus is not being repeated. Yet, the addition of this overtly associative or recollective component to the recognition memory task did not increase hippocampal activity.

It remains possible that the introduction of additional associative or recollective components to the recognition task might reveal greater hippocampal activity than was observed here in the Object-Object and Object-Word tasks. If conditions could be found that increase hippocampal activity in recognition memory tests, it will be important to distinguish effects due to the associative or recollective aspects of recognition from effects due to differences in performance or increases in the amount of material being remembered.

One might also consider the possibility that the failure to observe greater hippocampal activity in the Object-Word task than in the Object-Object task results from the fact that both tasks engaged a process of recollective search. In this case, hippocampal activity would be determined by whether recollective retrieval was being attempted, rather than by whether the retrieval attempt was successful. Previous studies have associated retrieval effort and retrieval success with the frontal lobes and the medial temporal lobes, respectively (e.g., Schacter et al. 1996). Moreover, the finding of greater activity for Hits than for Correct Rejections in all three tasks seems inconsistent with the idea that retrieval effort is the determining factor. If retrieval effort were the main factor, it is difficult to see why the activity associated with hits should have consistently exceeded the activity associated with correct rejections.

We suggest that all declarative memory tasks require some form of associative memory. Even the traditional recognition task requires that an association be made between what is presented for study and the context in which it is learned. It is this learning of relations, and learning about

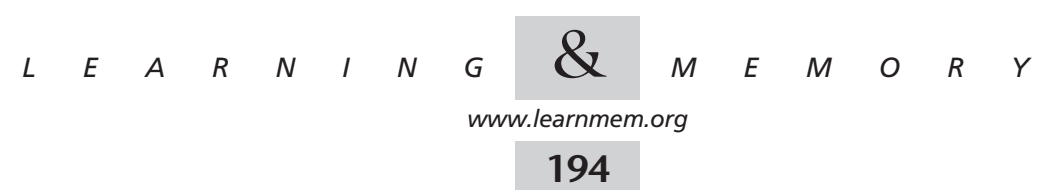


objects in contexts, that is thought to be at the heart of declarative memory and central to much of current thinking about the functions of the medial temporal lobe (Suzuki and Eichenbaum 2000; Zola and Squire 2000). This is not to say that the medial temporal lobe is equipotential with respect to memory functions. The anatomical organization of the medial temporal lobe has long suggested differentiation in the computational properties of its component structures. In particular, the hippocampal region is positioned at the end of a hierarchy of structures in the medial temporal lobe (for review, see Suzuki 1996) and seems ideally suited to the rapid storage of arbitrary patterns of activity (Marr 1971; Treves and Rolls 1994; O'Reilly and Rudy 2000). These ideas suggest that the hippocampal region is a general, crossmodal memory structure that combines and extends the processing of structures in the parahippocampal gyrus that are located earlier in the processing hierarchy. Although it remains possible that some declarative memory tasks may rely more heavily than other tasks on the extended processing capabilities provided by the hippocampal region, it seems likely that recall memory, recognition memory, episodic memory, semantic memory, and indeed all of declarative memory relies on and benefits from the processing afforded by the hippocampal region.

\section{MATERIALS AND METHODS}

Fourteen healthy volunteers ( 3 men, 11 women; mean age 27.8 years; range, 23-38 years) gave written informed consent prior to participating in the study. Across three different scanning sessions (separated by about one week), each participant was tested in all three task conditions: Object-Object, Word-Word, and ObjectWord. In the Object-Object task, line drawings of common nameable objects (Snodgrass and Vanderwardt 1980) were presented at both study and at test. In the Word-Word task, nouns (names of objects) were presented at both study and at test. In the ObjectWord task, pictures of nameable objects were presented at study, and nouns (names of objects) were presented at test. The order of the three tasks was randomized across participants.

Prior to each scanning session, participants viewed 80 stimuli (objects or words) twice each (duration, $2 \mathrm{sec}$; intertrial interval (ITI), $0.5 \mathrm{sec}$ ) outside the scanner with instructions to study the items for a later test. Approximately $30 \mathrm{~min}$ after this study phase, 240 items (the 80 studied target items, 80 nonstudied foil items, and 80 "null" items consisting of a blank screen) were presented (duration, $1.5 \mathrm{sec}$; ITI. $0.5 \mathrm{sec}$ ) while fMRI data were collected. Using their right hand, participants pressed one button on a response box to indicate that the item was a target item (or in the case of the Object-Word task to indicate that the item named a target item) and another button to indicate that the item was a foil item that had not been studied. During "null" trials, no stimuli were presented, and participants made no response. Which items served as targets and foils were counterbalanced across participants. Target trials, foil trials, and "null" trials were presented in a predetermined pseudo-random order (Dale 1999; Friston et al. 1999). The recognition task was administered three times in succession to each participant using the same stimuli but a different pseudorandom order.

Five of the fourteen participants did not have a full comple- ment of nine fMRI datasets (three recognition tests $\times$ three sessions). One participant was able to remain in the scanner for only two of the three recognition tests in each condition. For four other participants, some data were lost due to equipment difficulties. In all, 117 of 126 fMRI runs were available for analysis.

\section{Imaging Parameters}

Imaging was performed on a Siemens (Erlangen, Germany) $1.5 \mathrm{~T}$ Vision clinical MRI scanner equipped with a large clinical Aflex, coil and a bite bar. Functional T2*-weighted images were acquired using an echoplanar, single-shot pulse sequence with a matrix size of $64 \times 64$, TE of $43 \mathrm{msec}$, flip-angle of $90^{\circ}$, and an in-plane resolution of $4 \times 4 \mathrm{~mm}$. For each scanning run, 250 oblique axial images were acquired for each of 16 4-mm-thick slices aligned with the principle axis of the hippocampus (as determined by a series of sagittal localizer MRI scans for each participant). Images were acquired in an interleaved fashion with a TR of 2 sec. Stimulus presentation began on the fifth image and ended on the 244th image, allowing for the initial stabilization of the MR signal (four images) and for its return to baseline at the end of the task (six images). After the last fMRI scan, a high-resolution $\left(1 \mathrm{~mm}^{3}\right)$ MP-RAGE structural scan was acquired for anatomical localization.

\section{Image Analysis}

Images were first coregistered through time using a three-dimensional (3D) registration algorithm (Cox 1996). Within each run, voxels were eliminated if the signal magnitude changed more than $8 \%$ between two samples or if the mean signal level was below a threshold defined by the inherent noise in the data acquisition. Such voxels (when inside the brain) are likely to be contaminated by motion artifacts, venous effects, MR susceptibility, and distortion effects, or exceptionally poor signal-to-noise ratios, and cannot be considered to contain reliable data. Images were then spatially smoothed using a two-dimensional (in-plane) Gaussian kernel (full width half-maximum $=1.5$ voxels).

The behavioral data from each test were then scored, coding each trial as a Hit (correct "yes" response) a Miss (incorrect "no" response), a Correct Rejection (correct "no" response), a False Alarm (incorrect "yes" response), or a Null (no stimulus). A general linear model (GLM) of the activity in each voxel was then constructed using vectors coding for Hits, Misses, Correct Rejections, and False Alarms, in addition to six vectors coding the amount of 3D motion detected during image registration and terms for first and second order drift in the MR signal. The GLM was constructed using a deconvolution technique (D. Ward, "Deconvolution Analysis of FMRI Time Series Data," http://afni.nimh.nih.gov/afni) that first estimates the impulse response function within each voxel (based on six time points, $0-12 \mathrm{sec}$ ) and then performs a multiple linear regression. The sum of two time points (4-8 sec after the onset of the stimulus) corresponding to the expected peak in the hemodynamic response (the sum of the activity or the "area under the curve" during this time interval) was taken as the model's estimate of the response to each trial type. Three linear contrasts were calculated: Hits versus Correct Rejections, Hits versus Baseline, and Correct Rejections versus Baseline (Baseline activity estimated from the Null trials).

A nine-parameter transformation matrix was calculated to transform (Collins et al. 1994) the structural MRI from each participant's first scanning session to conform to the atlas of Talairach and Tournoux (1988). In addition, a six-parameter (rigid-body) transformation matrix was calculated to align each participant's

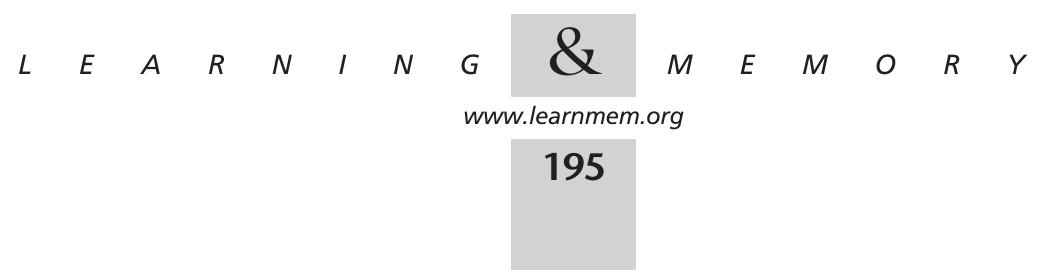


structural MRIs from the subsequent two scanning sessions to the scans from the first scanning session. The linear contrast terms (e.g., activity in a voxel for Hits vs. Correct Rejections for an individual participant and within an individual test) were divided by the mean signal level (to calculate a percent change), then transformed and resampled to $2.5 \mathrm{~mm}^{3}$ using the combination of the above two transformation matrices. Group analyses were conducted by performing voxel-wise t-tests on the linear contrast terms, setting an alpha-threshold of $P<.01$ for each voxel and a minimum cluster size of $125 \mathrm{~mm}^{3}$ of such voxels ( 8 resampled $2.5 \mathrm{~mm}^{3}$ voxels). For the analysis of Hits versus Correct Rejections, the t-tests were two-tailed versus zero. For the cross-run and crosstask analyses (e.g., Correct Rejections in Test 1 vs. Test 2 or Hits in Object-Object vs. Object-Word), the t-tests were pairwise (withinparticipant).

In addition to analyzing the data by using automated wholebrain alignment techniques (transforming to the atlas of Talairach and Tournoux 1988), the data were also analyzed using a crossparticipant alignment technique that specifically targeted the hippocampus. The extent of the hippocampal region was defined anatomically for each participant using the structural MRI from the first scanning session. Using the transformation matrix from the wholebrain alignment to provide an initial fit, an additional seven-parameter transformation matrix was calculated to transform each participant's hippocampal region onto the hippocampal region of a single representative participant. In this way, one can fine-tune the alignment of brains across participants so that it is selective for the hippocampal region and the neighboring structures (e.g., the parahippocampal gyrus) at the expense of more distant structures (e.g., occipital cortex). Within the hippocampal region, the results using this technique were qualitatively the same as those obtained with the whole-brain alignment technique. For clarity, and in order to interpret activity outside of the hippocampal region, only the results for the automated whole-brain alignment technique are presented here.

\section{ACKNOWLEDGMENTS}

We thank Shauna Stark, Jennifer Frascino, and Joyce Zouzounis for their assistance with data collection. This research was supported by the Medical Research Service of the Department of Veterans Affairs, National Institute of Mental Health Grants MH24600 and MH12278, the National Alliance for Research in Schizophrenia and Depression (NARSAD), and the Metropolitan Life Foundation.

The publication costs of this article were defrayed in part by payment of page charges. This article must therefore be hereby marked "advertisement" in accordance with 18 USC section 1734 solely to indicate this fact.

\section{REFERENCES}

Aggleton, J.P. and Brown, M.W. 1999. Episodic memory, amnesia, and the hippocampal-anterior thalamic axis. Behav. Brain Sci. 22: 425-444.

Brewer, J.B., Zhao, Z., Glover, G.H., and Gabrieli, J.D.E. 1998. Making memories: Brain activity that predicts whether visual experiences will be remembered or forgotten. Science 281: 1185-1187.

Brown, M.W. and Aggleton, J.P. 2001. Recognition memory: What are the roles of the perirhinal cortex and hippocampus? Nat. Rev. Neurosci. 2: 51-61.

Collins, D.L., Neelin, P., Peters, T.M., and Evans, A.C. 1994. Automatic 3D inter-subject registration of MR volumetric data in standardized Talairach space. J. Comput. Assist. Tomogr. 18: 192-205.

Cox, R.W. 1996. AFNI: Software for analysis and visualization of functional magnetic resonance neuroimages. Comp. Biomed. Res. 29: 162-173.
Dale, A.M. Optimal experimental design for event-related fMRI. 1999. Hum. Brain. Mapp. 8: 109-114.

Dale, A.M. and Buckner, R.L. 1997. Selective averaging of rapidly presented individual trials using fMRI. Hum. Brain. Mapp. 5: 329-340.

Eldridge, L.L., Knowlton, B.J., Furmanski, C.S., Bookheimer, S.Y., and Engel, S.A. 2000. Remembering episodes: A selective role for the hippocampus during retrieval. Nat. Neurosci. 3: 1149-1152.

Fernandez, G., Weyerts, H., Schrader-Bolsche, M., Tendolkar, I., Smid, H.G., Tempelmann, C., Hinrichs, H., Scheich, H., Elger, C.E., Mangun, G.R., et al. 1998. Successful verbal encoding into episodic memory engages the posterior hippocampus: A parametrically analyzed functional magnetic resonance imaging study. J. Neurosci. 18: $1841-1847$.

Friston, K.J., Zarahn, E, Josephs, O., Henson, R.N., and Dale, A.M. 1999 Stochastic designs in event-related fMRI. Neuroimage 10: 607-619.

Gabrieli, J.D.E, Brewer, J.B., Desmond, J.E., and Glover, G.H. 1997. Separate neural bases of two fundamental memory processes in the human medial temporal lobe. Science 11: 264-266.

Haxby, J.V., Ungerleider, L.G., Horwitz, B., Maisog, J.M., Rapoport, S.I., and Grady, C.L. 1996. Face encoding and recognition in the human brain. Proc. Natl. Acad. Sci. 93: 922-927.

Henke, K., Buck, A., Weber, B., and Wieser, H.G. 1997. Human hippocampus establishes associations in memory. Hippocampus 7: 249-256.

Henke, K., Weber, B., Kneifel, S., Wieser, H.G., and Buck, A. 1999. Human hippocampus associates information in memory. Proc. Natl. Acad. Sci. 96: $5884-5889$.

Kelly, W., Miezin, F., McDermott, K., Buckner, R.L., Raichel, M.E., Cohen, N.J., Ollinger, J.M., Akbudak, E., Contura, T.E., Snyder, A.Z., et al. 1998. Hemispheric specialization in human dorsal frontal cortex and medial temporal lobe for verbal and nonverbal memory encoding. Neuron 20: 927-936.

Lepage, M., Habib, R., and Tulving, E. 1998. Hippocampal PET activations of memory encoding and retrieval: The HIPER model. Hippocampus 8: 313-322.

Marr, D. Simple memory: A theory for archicortex. 1971. Philos. Trans. R. Soc. Lond. B. Biol. Sci. 262: 23-81.

Martin, A., Wiggs, C.L., Weisberg, J. 1997. Modulation of human medialtemporal lobe activity by form, meaning, and experience. Hippocampus 7: 587-593.

Milner, B., Squire L.R., and Kandel E.R. 1998. Cognitive neuroscience and the study of memory. Neuron 2: 445-468.

Mishkin, M., Suzuki, W.A., Gadian, D.G., and Vargha-Khadem, F. 1997. Hierarchical organization of cognitive memory. Philos. Trans. R. Soc. Lond. B. Biol. Sci. 352: 1461-1467.

O'Reilly, R.C. and Rudy, J.W. 2000. Computational principles of learning in the neocortex and hippocampus. Hippocampus 10: 389-397.

Schacter, D.L., Alpert, N.M., Savage, C.R., Rauch, S.L., and Alpert, M.S. 1996. Conscious recollection and the human hippocampal formation: Evidence from positron emission tomography. Proc. Natl. Acad. Sci. 93: 321-325.

Schacter, D.L. and Wagner, A.D. 1999. Medial temporal lobe activations in fMRI and PET studies of episodic encoding and retrieval. Hippocampus 9: 7-24.

Snodgrass, J.G. and Vanderwart, M. 1980. A standardized set of 260 pictures: Norms for name agreement, image agreement, familiarity, and visual complexity. J. Exp. Psychol. [Hum. Learn.] 6: 174-215.

Stark, C.E.L. and Squire, L.R. 2000a. Functional magnetic resonance imaging (fMRI) activity in the hippocampal region during recognition memory. J. Neurosci. 20: 7776-7781.

Stark, C.E.L. and Squire, L.R. 2000b. fMRI activity in the medial temporal lobe during recognition memory as a function of study-test interval. Hippocampus 10: 329-337.

Stern, C.E., Corkin, S., Gonzalez, R.G., Guimaraes, A.R., Baker, J.R., Jennings, P.J., Carr, C.A., Sugiura, R.M., Vadantham, V., and Rosen, B.R. 1996. The hippocampal formation participates in novel picture encoding: Evidence from functional magnetic resonance imaging. Proc. Natl. Acad. Sci. 93: 8600-8665.

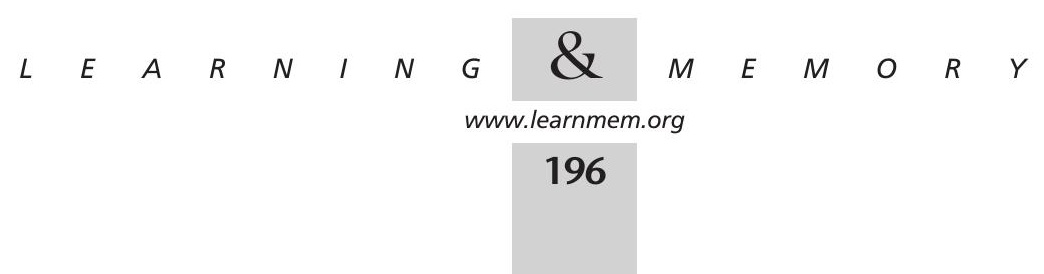


Suzuki, W. 1996. The anatomy, physiology and functions of the perirhinal cortex. Curr. Opin. Neurobiol. 6: 179-186.

Suzuki, W.A. and Eichenbaum, H. 2000. The neurophysiology of memory. Ann. N Y Acad. Sci. 911: 175-191.

Talairach, J. and Tournoux, P. 1988. A co-planar stereotaxic atlas of the buman brain. Thieme Medical, New York.

Treves, A. and Rolls, E.T. 1994. Computational analysis of the role of the hippocampus in memory. Hippocampus 2: 189-200.

Tulving, E. 1985. Memory and consciousness. Can. J. Psychol. 26: 1-12.

Vargha-Khadem, F., Gadian, D.G., Watkins, K.E., Connelly, A., Van Paesschen, W., and Mishkin, M. 1997. Differential effects of early hippocampal pathology on episodic and semantic memory. Science 277: 376-380.
Wagner, A.D., Gabrieli, J.D.E., and Verfallie, M. 1997. Building memories: Remembering and forgetting of verbal experiences as predicted by brain activity. Science 281: 1188-1191.

Zola, S.M. and Squire, L.R. 2000. The medial temporal lobe and the hippocampus. In The Oxford Handbook of Memory (eds. E. Tulving and F.I.M Craik), pp. 501-520. Oxford University Press, New York, NY.

Zola-Morgan, S.M. and Squire, L.R. 1993. Neuroanatomy of memory. Annu. Rev. Neurosci. 16: 546-563.

Received April 24, 2001; accepted in revised form July 10, 2001 


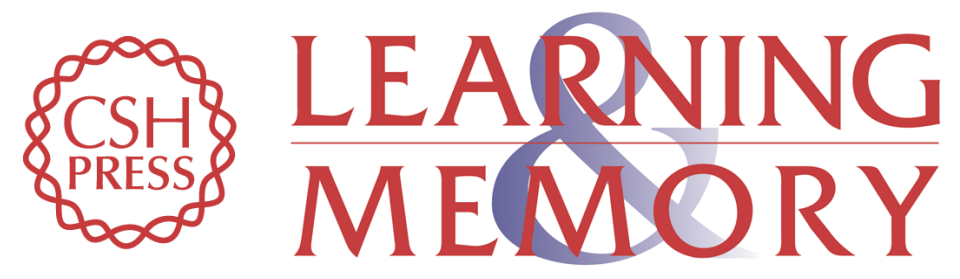

\section{Simple and Associative Recognition Memory in the Hippocampal Region}

Craig E.L. Stark and Larry R. Squire

Learn. Mem. 2001, 8:

Access the most recent version at doi:10.1101//m.40701

References This article cites 31 articles, 8 of which can be accessed free at: http://learnmem.cshlp.org/content/8/4/190.full.html\#ref-list-1

License

Email Alerting Receive free email alerts when new articles cite this article - sign up in the box at the Service top right corner of the article or click here. 